\title{
Orignial
}

\section{Surgical Findings \& Audiological Outcomes of Stapedotomy in Patients with Otosclerosis}

\author{
Mohammod Delwar Hossain ${ }^{1}$, Md. Rafiquzzaman², Nasir Uddin Ahamed ${ }^{3}$, \\ AKM Asaduzzaman', Md. Tauhidul Islam ${ }^{4}$, Muhammad Ali Azad ${ }^{4}$
}

\begin{abstract}
Objective: This study was focused on the assessment of patient's improvement in hearing, as measured by pure tone audiometry after stapedotomy for primary otosclerosis.

Materials and Methods: This prospective clinical study was performed in a total of 50 patients diagnosed with Primary Otosclerosis, who underwent Stapedotomy at the Department of ENT \& Head-Neck Surgery, Combined Military Hospital, Dhaka between June 2012 to January 2014. All patients were evaluated as per the candidacy criteria for stapedotomy and selected patients underwent surgery during the study period and were followed up for a period of 12 months in the Otology clinic. Pre-operative and Post-operative audiometric evaluation was done using conventional pure tone audiometry with standard calibrations. Post-operative audiometry was performed at 03 month, 06 months and 12 months. The bone-conduction \& air conduction thresholds and the AirBone gap (ABG), were documented and analysed at $0.5 \mathrm{KHz}, 1 \mathrm{KHz}, 2 \mathrm{KHz}$ and $4 \mathrm{KHz}$ frequencies respectively. The subjective outcomes in hearing were also recorded with a patient satisfaction questionnaire to assess improvement in quality of hearing after stapedotomy.
\end{abstract}

Results: Overall, the frequency specific pre-operative mean averaged Air Conduction thresholds were $63.3 \mathrm{~dB}$ at $500 \mathrm{~Hz}, 57.5 \mathrm{~dB}$ at $1 \mathrm{KHz}, 55.1 \mathrm{~dB}$ at $2 \mathrm{KHz}$ and $45.7 \mathrm{~dB}$ at $4 \mathrm{KHz}$. The frequency specific post-operative mean averaged Air conduction thresolds were $34.6 \mathrm{~dB}$ at $500 \mathrm{~Hz}, 28.9 \mathrm{~dB}$ at $1 \mathrm{Kz}$, $30.5 \mathrm{~dB}$ at $2 \mathrm{Kz}$ and $33.3 \mathrm{~dB}$ at $4 \mathrm{Kz}$. The frequency specific mean average post operative Air Bone Gap (ABG) closure was achieved by $28.7 \mathrm{~dB}$ at $500 \mathrm{~Hz}, 27.6 \mathrm{~dB}$ at $1 \mathrm{KHz}, 24.6 \mathrm{~dB}$ at $2 \mathrm{KHz}$ and $12.4 \mathrm{~dB}$ at $4 K \mathrm{Kz}$ by the time of completion of the study at 01 year. A successful closure of $A B$ gap to less than $10 \mathrm{~dB}$ was achieved in the speech frequencies of $2 \mathrm{KHz}$ and $4 \mathrm{KHz}$ in $84 \%$ of cases. Overall, the frequency specific bone-conduction thresholds were unchanged postoperatively in all cases except one, showing that no significant sensori-neural impairment had occured due to the stapedotomy procedure. 1 patient developed post-op severe vertigo which was self-limiting after six weeks. A few interesting cases with anomalous intra-operative findings were also documented and reported herewith. These included monopodal stapes ( $n=1)$, anomolous facial nerve $(n=1)$, laterally placed chordatympani nerve ( $n=1)$, high jugular bulb, Malleus ankylosis $(n=1)$ and persistent stapedial artery $(n=1)$. All patients included in our study had significant subjective audiological improvement and responded satisfactorily to the questionnaire formulated to assess their hearing quality after stapedotomy.

1. Lt. Col. Classified ENT Specialist \& head-Neck Surgeon, Department of ENT \& Head-Neck Surgery, $\mathrm{CMH}$, Dhaka.

2. Brig. Gen. Adviser Specialist in ENT \& Head-Neck Surgery, CMH, Dhaka.

3. Brig. Gen. Commandant, $\mathrm{CMH}$, Dhaka Cantonment.

4. Lt. Col. Classified ENT Specialist, CMH, Dhaka.

Address for Correspondence: Lt. Col. Mohammod Delwar Hossain, Department Of ENT \& headNeck Surgery, Combined Military Hospital, Dhaka Cantonment, Dhaka 1206, E-mail:delwar1064.bd @gmail.com 
Conclusions: Our case study confirms that stapedotomy is a safe and successful procedure providing long-term hearing improvement in primary otosclerosis. Obliterative otosclerosis, biscuit or floating footplate, monopodal stapes, anomalous facial nerve and persistent stapedial artery may be special scenarios encountered during stapedotomy and they need judicious management by an experienced surgeon. Our study shows that meticulous selection of cases for stapedotomy will result in highly successful audiological outcomes.

Key words: Primary Otosclerosis, Stapedotomy, Teflon piston, Carhart's notch, obliterative otosclerosis, persistent stapedial artery.

\section{Introduction}

Stapedotomy surgery is safe and efficacious in the hands of an experienced otologist and audiological outcomes are often adjudged based upon this surgical acumen. Minor issues like post op vertigo and tinnitus needs to be adressed in detail during counselling for stapedotomy in order to provide comprehensive patient awareness in preparation for surgery.

Otosclerosis is a primary disease of the labyrinth bone capsule consisting of one or more localized foci in which bone resorption and deposition take place repeatedly. This focus may gradually invade the annular ligament and the stapes, causing bony ankylosis and deterioration of the air conduction of sound. Treatment of otosclerosis can be medical, surgical or hearing aid support but undoubtedly the surgical treatment of otosclerosis is the most preferred option. Sodium fluoride (NaF) and biphosphonates are the two most commonly used substances for medical treatment of otosclerosis. In practice, an intervention should only be considered in cases with deafness over $30 \mathrm{~dB}$ and a Rinne value in this order, although the possibility of recovery by bone conduction (Carhart effect) can and should be taken into account. The short-term results are undoubtedly very good in the recent literature.

\section{Material and Method}

This prospective clinical study was performed in a total of 50 patients diagnosed with
Primary Otosclerosis who underwent Stapedotomy at the Department of ENT \&Head-neck Surgery, Combined Military Hospital, Dhaka between June 2012 to January 2014. All patients suspected to have Otosclerosis, were evaluated as per the candidacy criteriae for stapedotomy and selected patients underwent surgery during the study period and were followed up for a period of 1 year in the Otology clinic. Preoperative and Post-operative audiometric evaluation was done using conventional pure tone audiometry. Post-operative audiometry was performed at 3 month, 6 months and 1 year. The bone-conduction and air conduction thresholds and the Air-bone gap (ABG), were assessed at $0.5 \mathrm{KHz}, 1 \mathrm{KHz}, 2 \mathrm{KHz}$ and 4 $\mathrm{KHz}$ frequencies. The subjective outcomes were also recorded with a patient satisfaction questionnaire to assess improvement in quality of life after stapedotomy.

We conducted a prospective clinical study in 50 patients operated on for stapedotomy in the period from June 2012 to January 2014 at our hospital. The surgical technique used in all patients was stapedotomy. The basic technique was performed under local anaesthesia and deep sedation, by common transmeatal approach and incudo-stapedial disarticulation, dissection of the stapes tendon, fracture of posterior crus of stapes and perforation of the foot plate with a skeeter drill. Prostheses used were $0.6 \mathrm{~mm}$ diameter Teflon piston of appropriet length. Fenestrum sealed with ear lobule fat autograft after the placement of the prosthesis. 


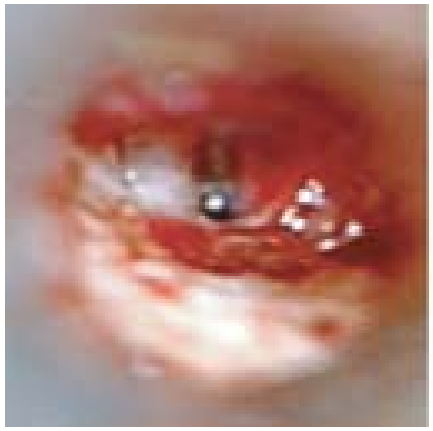

Fenestration on posterior middle $3^{\text {rd }}$ of footplate

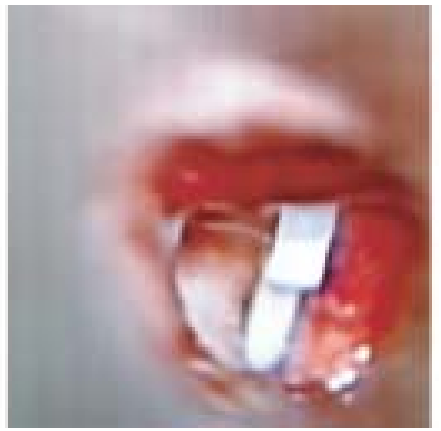

$0.6 \mathrm{~mm}$ Teflon piston in situ.

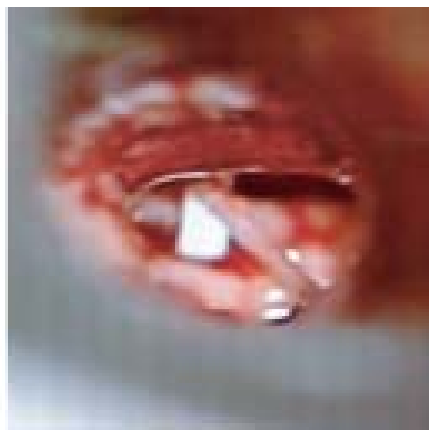

Corda tympani nerve is anchored to prevent displacement.

Fig.-1: Stapedotomy procedure

Few special anatomical rare scenarios were obsereved during surgery. 1 Patient had monopodal stapes, 1 malleus ankylosis, 1 very high facial nerve, 1 high jugular bulb and 1 had persistent stapedial artery those were encountered very carefully.

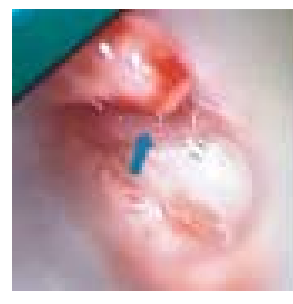

Intra-operative Otomicroscopi View of Persistent Stapedial Artery

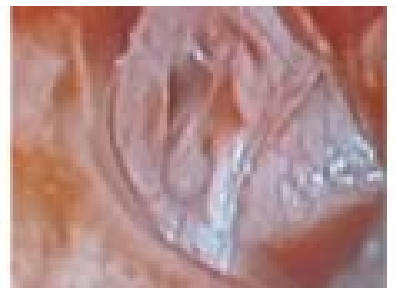

High facial nerve

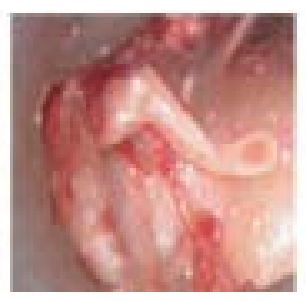

Malleus ankylosis

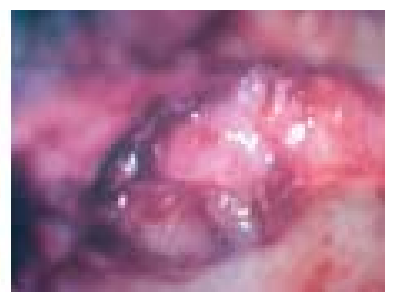

High jugular bulb

Fig 2: Intra op special anatomical variations

Audiometric Assessment included the preoperative and postoperative air-bone gap (ABG), air-conduction (AC) thresholds and bone-conduction (BC) thresholds were done at 3 months, 6 months and 12 months after surgery. $A C$ and $B C$ results that were obtained pre and post operatively, used for calculation of ABG and pure-tone averages (PTAs). We used a four-frequency PTA for $A C$ and BC thresholds $(0.5,1,2$, and $4 \mathrm{kHz})$ obtained at the last follow-up visit.

\section{Statistical analysis}

The results were recorded in an Excel spreadsheet which was then processed using the SPSS statistics package for Windows. The descriptive analysis of quantitative variables was performed by determining the mean and standard deviation for continuous variables and absolute \& relative frequencies for categorical variables. The comparative analysis was performed using the nonparametric Friedman test (Friedman analysis of variance by ranks), taking into account the audiometric results in the 3 periods. The accepted level of statistical significance in our work is $P=.05$.

\section{Results}

All 50 operated ears have been included in this study; 27 (54\%) in women and 23 (46\%) in men. The age range is $20-63$ years with a mean of 36 years. $60 \%$ of patients were younger than 40, 30\% were between 41 and 49 , and $10 \%$ were over 50 years of age at the time of the intervention. The most common 


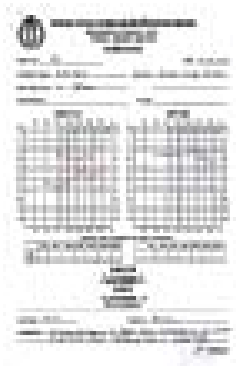

Pre op

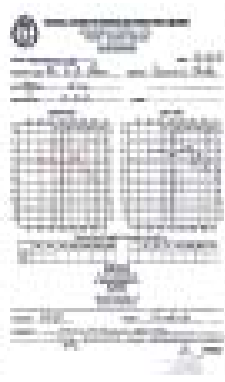

Post op

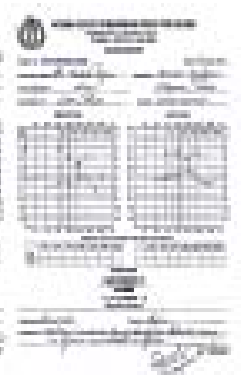

Pre op

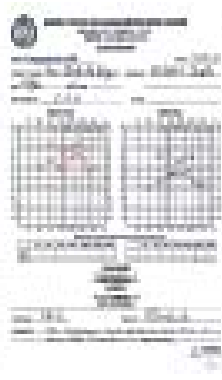

Post op

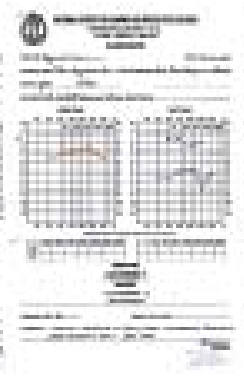

Pre op

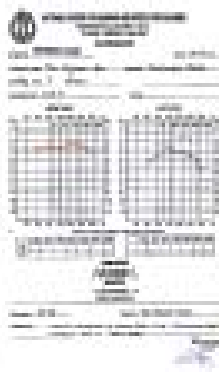

Post op

Fig.-3: Pre \& Post op PTA of Few patients

clinical presentation among our patients was hearing loss (80\%), followed by a combination of hearing loss and tinnitus (48\%). Less commonly, we encountered the combined presentation of hearing loss \& giddiness (4\%) and the combination of hearing loss, giddiness \& tinnitus, which was only observed in $2 \%$ of patients.

We noted that there were more operations on the right ear in 31 cases (62\%) than on the left 19 cases (38\%).

Post-operative audiometry was conducted in an interval of 3 months, 6 months and 12 months after surgery.

Overall, the postoperative $A B G$ closure was closed to $10 \mathrm{~dB}$ in $42(84 \%)$ cases. The individual frequency specific postoperative ABG closure was $28.7 \mathrm{~dB}, 27.6 \mathrm{~dB}, 24.6 \mathrm{~dB}$ and $12.4 \mathrm{~dB}$ respectively. The frequency specific bone-conduction thresholds were unchanged postoperatively.

\section{Table-I}

Mean average Air Conduction Thresholds pre op and post op at 3 month, 6 month and 1year $(N=50)$

\begin{tabular}{|c|c|c|c|c|}
\hline$\overline{\text { PTA }}$ & $\begin{array}{l}\text { Pre op } \\
\text { (dBHL) }\end{array}$ & $\begin{array}{c}\text { Post-op } \\
\text { (3 month) } \\
\text { (dBHL) }\end{array}$ & $\begin{array}{c}\text { Post-op } \\
(6 \text { months) } \\
(\mathrm{dBHL})\end{array}$ & $\begin{array}{r}\text { Post-op } \\
\text { (1 year) } \\
\text { (dBHL) }\end{array}$ \\
\hline $500 \mathrm{~Hz}$ & 63.3 & 45.2 & 38.3 & 34.6 \\
\hline $1 \mathrm{KHz}$ & 57.5 & 39.4 & 31.6 & 28.9 \\
\hline $2 \mathrm{KHz}$ & 55.1 & 37.7 & 32 & 30.5 \\
\hline $4 \mathrm{KHz}$ & 45.7 & 34.8 & 34.2 & 33.3 \\
\hline
\end{tabular}

A significant postoperative sensorineural hearing loss (SNHL) was seen in $1(2 \%)$ of case in this series. 1 patient developed post op persistent vertigo which subsided over time.

\section{Discussion}

As for the airborne route, the short-term results are, without question, very good; Birch et $\mathrm{al}^{1}$ showed in their study a gain of between 25 and $35 \mathrm{~dB}$ on average for the air route.

Previous reports of otosclerosis surgery have shown that stapedotomy gives better highfrequency gain and reduces the risk for sensorineural hearing loss (SNHL). ${ }^{2,4}$

In a study presented by Spandaw et $\mathrm{al}^{3}$ the DHT descended from $36 \mathrm{~dB}$ before surgery to $11 \mathrm{~dB} 1$ year after surgery, only to rise again up to $17 \mathrm{~dB} 10$ years after surgery.

The symptom that most often affects patients with otosclerosis is hypoacusis, although the main reason for consultation is often tinnitus ${ }^{5,6}$. In a study presented by del Bo et al, ${ }^{4} 56 \%$ of patients had tinnitus before surgery. In our study, the figures are somewhat lower, $48 \%$ of our patients subjected to surgery. Vertigo is a common symptom in patients operated on for otosclerosis. ${ }^{5,} 6$ In their study, Sedwick et al 7 found that $23.3 \%$ of post-surgical patients presented this symptom after stapedectomy 
or stapedotomy. Our study found occasional vertigo in $6(12 \%)$ of patients and 1 patient developed persistant vertigo subsided after 06 weeks. Prosthesis dislocation, migration out of the oval window fenestra, and complete incus erosion were the most common causes for failure in their series.

Several studies have commented on the variable of patient age at the time of stapes surgery ${ }^{7}, 8$, Stapedectomy in seniors is controversial. It is indicated to avoid the use of a hearing aid or to facilitate hearing aid fitting, when the association of otosclerosis and presbyacusis makes hearing aid provision unsatisfactory. ${ }^{9,10}$ In a series of 154 cases of stapedectomy in the elderly, Lippy et al ${ }^{11}$ stated that the percentage of patients (90.9\%) having a successful surgical result (postoperative $A B G$ within $10 \mathrm{~dB}$ ) was similar to younger patients in the comparison group with a successful surgical result (90\%). Our results compare favorably with previous series, and our low rate of complications also confirms that advanced age is not a contraindication to stapedotomy. Moreover, surgery will stop the progression of the disease and allow the patient better use of a hearing aid with an important improvement in sound discrimination. ${ }^{8}$

Table -II Mean average Air Bone Conduction Gap Closure Post Stapedotomy in $\mathrm{dBHL}(N=50)$

\begin{tabular}{lccc}
\hline Frequency & $\begin{array}{c}\text { R.Ramalingam } \\
\text { et al }{ }^{12}\end{array}$ & $\begin{array}{c}\text { EA myrvoll } \\
\text { et al }{ }^{9}\end{array}$ & $\begin{array}{c}\text { Our } \\
\text { study }\end{array}$ \\
\hline $500 \mathrm{~Hz}$ & 28.4 & 29.0 & 28.7 \\
$1 \mathrm{KHz}$ & 24.6 & 26.7 & 27.6 \\
$2 \mathrm{KHz}$ & 18.34 & 19.0 & 24.6 \\
$4 \mathrm{KHz}$ & 14.52 & 12.7 & 12.4 \\
\hline
\end{tabular}

In our study of 50 patients mean difference between pre and post op air conduction threshold (dbHL) was 28.7, 27.6, 24.6 and 12.4 at $0.5,1,2$ and $4 \mathrm{KHz}$ respectivly. (Table-1)

In one study by R.Ramalingam ${ }^{12}$ et al Mean difference between pre and post op air conduction threshold (dbHL) was 28.4, 24.6, 18.4, and 14.52 at $0.5,1,2$ and $4 \mathrm{KHz}$ respectivly. In another Study done by E A Myrvoll et al ${ }^{9}$ Mean difference between pre and post op air conduction threshold (dbHL) was 29, 26.7, 19, 12.7 at 0.5, 1, 2 and $4 \mathrm{KHz}$ respectivly. A significant postoperative sensorineural hearing loss (SNHL $>15 \mathrm{~dB}$ ) was seen in $0.5 \%$ of cases in a study by Robert vincent et al. ${ }^{10}$ In our study, the postoperative four-frequency $A B G$ was closed to $10 \mathrm{~dB}$ in $42(84 \%)$ compared with $23.58 \mathrm{~dB}$ preoperatively. The postoperative fourfrequency average $A C$ threshold was 31.82 dB compared with $55.4 \mathrm{~dB}$ preoperatively. Postoperatively, the four-frequency average BC threshold was $25.5 \mathrm{~dB}$ compared with 25.8 $\mathrm{dB}$ preoperatively (remains unchanged), and overclosure (post op improvement) occurred in $2(4 \%)$ cases.

90.9\% tinnitus patients reported improvement and $9.09 \%$ noted no change in tinnitus in a study conducted by Carlos A Oliveira ${ }^{5}$ While in our study $86 \%$ tinnitus patients reported improvement.

In study by Robert Vincent et al. ${ }^{10}$ the overall rate of failures was $6.6 \%$. There were $7.8 \%$ cases of severe postoperative SNHL. In our study, post op SNHL rate was in $1(2 \%)$ case.

\section{Conclusion}

Our series confirms that stapedotomy for otosclerotic stapes fixation is a safe and successful treatment for hearing improvement. Obliterative otosclerosis, biscuit footplate, monopodal stapes and persistent stapedial artery may be encountered during stapedotomy. Our study shows that judicious selection of cases for stapedotomy will result in high success rate 
in audiological outcomes in patients with otosclerosis. Stapedotomy surgery is safe and efficacious in the hands of an experienced otologist and audiological outcomes are often adjudged based upon this surgical acumen. Minor issues like post op vertigo and tinnitus needs to be adressed during counselling for stapedotomy in order to provide patients awareness in preparation for surgery.

\section{References}

1. Birch L, Elbrond O, Pedersen U. Hearing improvement after stapedectomy: Up to 19 years follow-up period. J Laryngol Otol. 1986; 100:1-7.

2. Fish U. Stapedotomy vs stapedectomy. Am J Otol 1982; 4:112Y7.

3. Spandow O, Soderberg O, Bohlin L. Long-term results in otosclerotic patients operated by stapedectomy and stapedotomy.Scand Audiol 2000; 29:186Y90.

4. del Bo MD, Zaghis A, Ambrosetti U. Some observations concerning 200 stapedotomies: fifteen years postoperatively. Laryngoscope. 1987; 97: 1211-3

5. Carlos A olivera.Long term follow up of tinnitus in patients with Otosclerosis after stapes surgery. The international tinnitus journal.2004;10(2):197-201

6. Smyth G, Hassard T, El Kordy A. Longterm hearing performance after the stapedectomy. J Laryngol Otol 1980; 94:1097Y105.

7. Sedwick J, Louden C, Shelton C. Stapedectomy vs stapedotomy.Do you really need a laser?Arch Otolaryngol Head Neck Surg 1997;123:177Y80

8. Proschel U, Jahnke K. Otosclerosis in the elderly. The effect of stapes-plasty on hearing ability and hearing aid management. HNO 1993; 41:77Y82.

9. E A Myrvoll, N.C Stenklev, E Laukli. Stapes surgery outcome evaluation. Arnold W, Hausler R: Otosclerosis and stapes surgery. Adv otolaryngol. Basel, Karger, 2007, vol 65, PP 340-342

10. Robert Vincent, Neil M. Sperling, John Oates, and Mudit Jindal.Surgical Findings and Long- term Hearing Results in 3,050 Stapedotomies for Primary Otosclerosis: A Prospective Study with the Otology-Neurotology Database. Otology \& Neurotology 27:S25YS47 _ 2006, Otology \& Neurotology, Inc

11. Lippy W, Burkey J, Fucci M, et al. Stapedectomy in the elderly. Am J Otol 1996; 17:831Y4.

12. Emmanuel Sara Kolo and R. Ramalingam. Hearing results in adults after stapedotomy. Niger Med J.2013 Jul-Aug; 54(4):236-239. 